\title{
Carotidynia in a Patient Receiving an Allogeneic Hematopoietic Cell Transplantation: A Case Report to Support a Disputed Entity
}

\section{Introduction}

Carotidynia was originally described by Fay in 1927 [T. Fay Arch Neurol Psychiatry 1927; 18:309-15] as an atypical neuralgia consisting of pain and tenderness at the level of the carotid bifurcation. It was classified by the International Headache Society (IHS) in 1988 as a self-limiting syndrome comprising unilateral neck pain, tenderness over the carotid bifurcation, and absence of structural abnormalities, but was then removed in 2004 as a distinct disease since further research indicated that it should be regarded as a syndrome encompassing other distinct disease processes in the carotid region. Case reports [F. Comacchio et al. Acta Otorhinolaryngol Ital. 2012; 32(4): 266-9] have since described similar clinical presentations of neck pain and tenderness over the carotid bifurcation of unknown etiology, where patients undergo spontaneous remission, and imaging studies often agree on findings of perivascular inflammation, luminal integrity, and normal blood flow in the affected carotid [N. Kosaka et al. Eur Radiol. 2007;17(9):2430-3] - supporting the existence of carotidynia as a distinct entity.

We present a case report of carotidynia in a patient undergoing an allogeneic hematopoietic cell transplantation (HCT) with non-myeloablative conditioning where ultrasound (US) examinations and computed tomography (CT) angiography revealed the above-mentioned findings.

\section{Case description}

A 47-year-old male patient with GATA2-mutation-associated myelodysplastic syndrome and pulmonary fibrosis was admitted for an HCT. He had successfully completed the conditioning regimen consisting of fludarabine $30 \mathrm{mg} / \mathrm{m}^{2}$ o.d. from day -4 to -2 and 2 Gy of total body irradiation o.d. on day -1 and 0 . Prophylaxis of graft-versushost disease (GVHD) and rejection consisted of tacrolimus $0.06 \mathrm{mg} / \mathrm{kg}$ b.i.d. starting on day -3 with a target concentration of 10-15 microgram/L the first 28 days post-transplant and mycophenolate mofetil $15 \mathrm{mg} / \mathrm{kg}$ given once $4-6 \mathrm{~h}$ after infusion of the graft and $15 \mathrm{mg} / \mathrm{kg}$ t.i.d. from day +1 .
Antibacterial prophylaxis consisted of ciprofloxacin $500 \mathrm{mg}$ b.i.d when the neutrophils were below $0.5 \times 10^{9} / \mathrm{L}$, and in this patient ciprofloxacin was started on day -1 as the total leukocyte count fell to $0.85 \times 10^{9} / \mathrm{L}$ (ref. $3.5-8.8 \times 10^{9} / \mathrm{L}$ ) on that day. Antifungal prophylaxis consisted of fluconazole $400 \mathrm{mg}$ o.d. from day 0 . On day +1 the antibiotic treatment was changed to ceftazidime $2 \mathrm{~g}$ given intravenously t.i.d. due to a rise in C-reactive protein (CRP), while the patient remained asymptomatic and afebrile during the period of leukopenia which reached a nadir of $0.11 \times 10^{9} / \mathrm{L}$ on day +7 . On day +12 the patient developed severe left-sided neck pain. Physical examination revealed a localized swelling of approximately $2 \times 2 \mathrm{~cm}$ anterior to the left sternocleid muscle with no concurrent findings in the mouth or throat, including no signs of mucositis. Ibuprofen $400 \mathrm{mg}$ was given once but proved ineffective and the patient was treated with oxycodone. Laboratory tests on the same day found a leucocyte count of $0.36 \times 10^{9} / \mathrm{L}$ - up from $0.29 \times 10^{9} / \mathrm{L}$ and CRP of $30 \mathrm{mg} / \mathrm{L}$ (ref. $<10 \mathrm{mg} / \mathrm{L}$ ), which rose to $1.1 \times 10^{9} / \mathrm{L}$ and $125 \mathrm{mg} / \mathrm{L}$, respectively, after two days. Antibiotic treatment was broadened to meropenem, vancomycin and caspofungin, and on day +17 the CRP level had fallen to $58 \mathrm{mg} / \mathrm{L}$ while the leukocyte count rose slightly to $1.5 \times 10^{9} / \mathrm{L}$.

US examination was requested due to suspicion of abscess formation. US on day +13 , where the total leukocyte count was $0.56 \times 10^{9} / \mathrm{L}$, revealed a thickened wall of the common and internal carotid artery on the left side with no intraluminal plaques as well as thickening of the periarterial con-

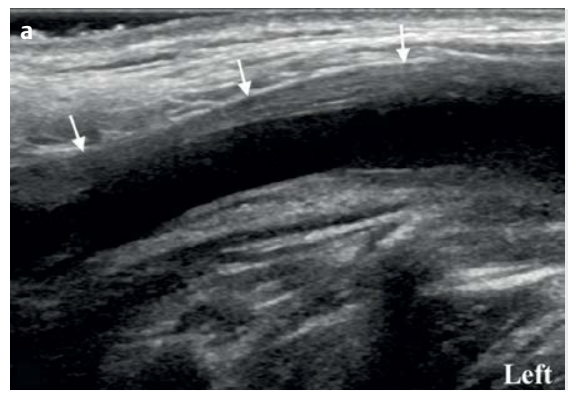

Fig. 1 a US examination showing a thickened left common carotid wall (arrows) and luminal integrity. b Power Doppler showing normal vascularity of the periarterial tissue

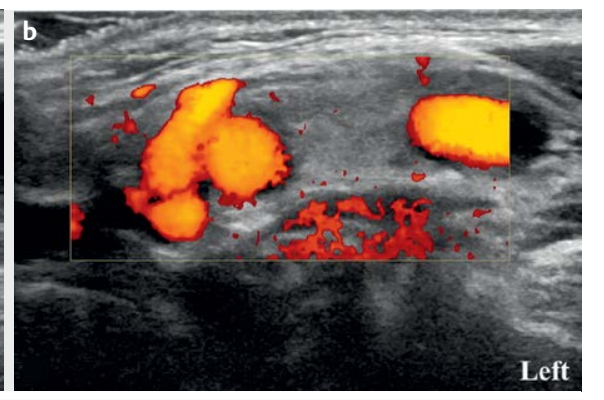

nective tissue ( $\triangleright$ Fig. 1 ). The intravascular flow was uncompromised and there was no hyperemia in the periarterial connective tissue. Interestingly, the common and internal carotid artery on the right side showed similar findings albeit to a lesser degree. CT angiography was subsequently performed on suspicion of vasculitis and revealed a slightly thickened periarterial connective sheath around the carotids, primarily on the left side ( $\mathbf{F i g}$. 2 ). The findings were interpreted as possible vasculitis with inflammation in the adjacent connective tissue.

The patient was broadly investigated for an infectious cause, including multiple blood cultures obtained while under continuous antibiotic therapy as well as blood tests and mouth swabs for viral and fungal origins, but all tests (including PCR for Epstein-Barr Virus, cytomegalovirus, influenza virus $A$ and $B$, parvovirus $B 19$ and respiratory syncytial virus) were negative.

28 days after transplantation, US examination including elastography ( $\triangleright$ Fig. $\mathbf{3}, \mathbf{4}$ ) revealed that the changes seen in the arteries and perivascular tissue had significantly diminished. The symptoms have ultimately remitted, and the episode was conclusively considered consistent with carotidynia.

\section{Discussion}

The complex nature of the presented case offers several potential explanations for the syndrome. A bacterial origin cannot be ruled out, especially in an immunosuppressed patient, but it seems unlikely given the broad antibiotic cover and the bilateral disease. Abscess formation with a leucocyte 
count below $1 \times 10^{9} / \mathrm{L}$ is furthermore considered improbable. A viral infection either as a primary infection or as a reactivation of a dormant virus - or a reactive disease following a viral infection - remains a possibility. The symptoms occurred at the same time as the patient experienced engraftment, and though there were no signs of acute GVHD or engraftment syndrome, an alloreactive response is also a possibility.

Ultrasound was performed as the firstline examination as it has very high spatial

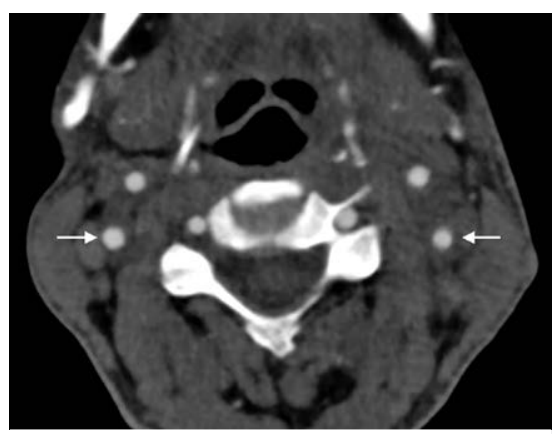

- Fig. 2 CT angiogram depicting the slightly thickened periarterial connective sheaths around the internal carotid arteries, primarily on the left side. resolution and is easy to perform bedside on an uncomfortable patient. It will also reveal the mentioned acute vascular causes of neck pain as well as demonstrate perivascular infiltration and possible flow abnormalities. At the same time US has the benefit of avoiding exposure to radiation and contrast agents.

$\mathrm{CT}$ and/or MRI remain relevant investigations to rule out vascular causes of neck pain such as arterial dissection or intraluminal hemorrhage. If inflammatory changes are present, MRI has the greater sensitivity in delineating the abnormally enhancing tissue [N. Kosaka et al. Eur Radiol. 2007; 17(9):2430-3]. In retrospect, MRI would have been the optimal modality in the presented case, but due to logistical challenges $C T$ angiography was performed.

In a recent review $[A$. Lecler et al. AJNR Am J Neuroradiol. 2017;38(7):1391-1398] including 47 patients with confirmed clinical carotidynia who all experienced full recovery, the syndrome was unilateral in 45 cases and bilateral in 2. Only 3 patients saw a mild increase in systemic inflammatory markers such as sedimentation rate or CRP. Interestingly, 8 patients had an autoim-
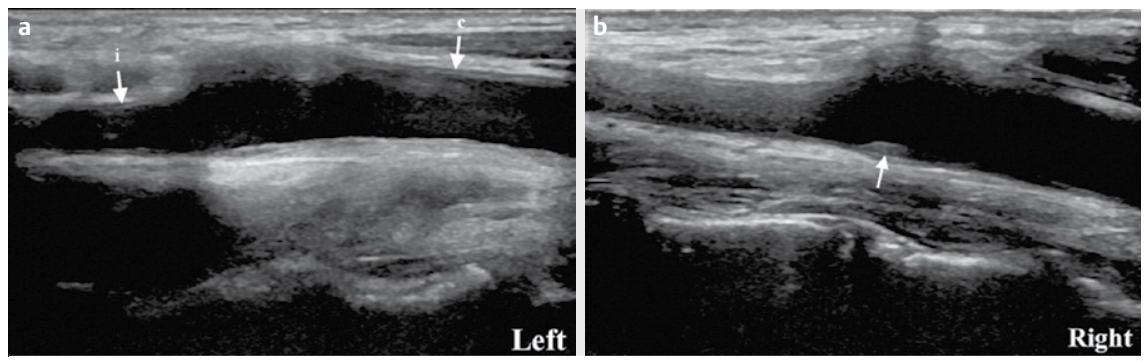

- Fig. 3 a Repeat US examination on day 28 showing minimally increased thickness of the vessel wall of the left common (c) and internal (i) carotid arteries. b US of the right carotid bifurcation reveals a little intimal plaque (arrow) but otherwise a normal vessel wall

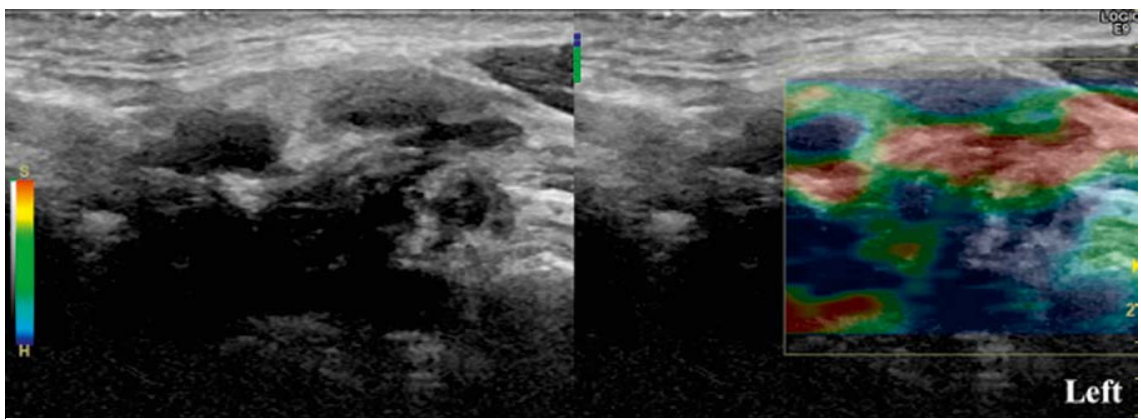

- Fig. 4 US elastography of the left carotid artery on day 28 showing slightly increased stiffness of parts of the periarterial tissue (in blue and green) at the level of the bifurcation mune disease such as rheumatoid arthritis or systemic lupus erythematosus, and 7 of these presented with relapse of their autoimmune disease simultaneously with the carotidynia. All patients except 4 presented with periarterial infiltration at the level of the carotid bifurcation, and mild lumen caliper narrowing was observed in 9 patients. No hemodynamic changes were observed in the 23 patients examined with color Doppler.

In a unique study, Upton et al. [PD. Upton et al. Otolaryngol Head Neck Surg. 2003;129(4):443-4] investigated the perivascular tissue in a patient with carotidynia who had undergone carotid endarterectomy. Microscopic examination revealed nonspecific inflammatory changes including vascular proliferation, proliferation of fibroblasts, and low-grade chronic active inflammation predominately involving lymphocytes. They did not find the changes to be consistent with abscess wall or granulation tissue, nor for them to be characteristic of inflammation around a large vessel in vasculitis.

The presented case is in alignment with the previously described cases of carotidynia with regards to the clinical presentation as well as imaging findings and remains a relevant differential diagnosis in acute neck pain. We find US to be a valuable investigation in patients presenting with carotidynia both as a primary examination as well as for follow-up.

\section{Conflict of Interest}

The authors declare that they have no conflict of interest.

Authors

Tobias Thostrup Andersen ${ }^{1}$, Martin Lundsgaard Hansen ${ }^{1}$, Søren Lykke Petersen ${ }^{2}$, Caroline Ewertsen ${ }^{3}$

Affiliations

1 Rigshospitalet, Department of Radiology, Kobenhavn, Denmark

2 Rigshospitalet, Department of Haematology, Kobenhavn, Denmark 
3 Copenhagen University Hospital, Rigshospitalet, Department of

Radiology, Copenhagen OE, Denmark

\section{Correspondence}

Dr. Tobias Thostrup Andersen, MD

Rigshospitalet, Department of Radiology

Blegdamsvej 9

Kobenhavn, 2100

Denmark

Tel.: $+45 / 35458726$

tobias.thostrup.andersen.01@regionh.dk
Bibliography

DOI https://doi.org/10.1055/a-0647-2330

Ultrasound Int Open 2018; 4: E106-E108

(c) Georg Thieme Verlag KG Stuttgart .

New York

ISSN 2199-7152

(ㄷ) (i) $($ 우 $\ominus$ 